\title{
Estudio transversal clínico y epidemiológico de la enfermedad de Chagas en una área rural del Nordeste Argentino
}

\author{
Clinical and epidemiological transversal study on Chagas' disease \\ in a rural area of the Argentinian Northeastern \\ María Esther Bar, Domingo Luis Pozzer, Benedicto Mobel Alvarez, \\ Julio Andrés Vallejos y Rubén Alberto Storino
}

\begin{abstract}
Resumen Esta investigación tiene por objeto analizar las características epidemiológicas, clínicas, electrocardiográficas y radiológicas de una población de seropositivos y seronegativos al Trypanosoma cruzi en una área rural del Departamento San Miguel, provincia de Corrientes, Argentina. Se seleccionaron 132 pobladores (58 hombres y 74 mujeres) de todos los grupos etareos para la realización de un examen clínico y cardiovascular. Se registraron síntomas y signos compatibles con afección cardíaca, tensión arterial, electrocardiograma de 12 derivaciones y radiografía anteroposterior de tórax. En la signo-sintomatología, anomalías electrocardiográficas y en las alteraciones radiográficas detectadas, no se observaron diferencias estadísticamente significativas entre seropositivos y seronegativos. Se concluye que si bien en la población objeto de estudio la infección chagásica no se asoció a mayor prevalencia de cardiopatía, cabe destacar que los pacientes chagásicos pertenecían a un grupo etareo muy joven con un 54,0\% de ellos menores de 20 años y que el 45,0\% de los pacientes mayores de 41 años tenían alteraciones electrocardiográficas.
\end{abstract}

Palabras-claves: Trypanosoma cruzi. Infección chagásica. Alteraciones electrocardiográficas y radiográficas.

\begin{abstract}
The main goal was to know the epidemiologic, clinical, electrocardiographic and radiologic characteristics among a population of seropositives and seronegatives to Trypanosoma cruzi in a rural area of the Departament of San Miguel, province of Corrientes, Argentina. One hundred and thirty-two patients of different ages: 2-79 years old were researched (58 males, 74 females). In order to make a thorough assessment clinical evaluation and cardiologic testing were carried out. Signs and symptoms consistent with heart disease, blood pressure, 12-lead ECG registry and chest $x$-ray (PA view) were registered. In signs and symptoms, abnormal ECG patterns and radiologic abnormalities, non-significant statistic difference were observed. Although we were unable to find a significant relationship between chagasic infection and a higher prevalence for heart disease, it is important to stress the fact that $54.0 \%$ of the studied population was largely composed of very young patients whose ages were less than 20 years old, and $45.0 \%$ of those older than 41 years showed ECG abnormalities.
\end{abstract}

Key-words: Trypanosoma cruzi. Chagasic infection. Electrocardiographic and radiological alterations.

\footnotetext{
Cátedra de Artrópodos. Facultad de Ciencias Exactas y Naturales y Agrimensura. Universidad Nacional del Nordeste, Instituto de Cardiología de Corrientes "Juana F. Cabral" y Centro de enfermedad de Chagas, Fundación INCALP de La Plata, Argentina. Trabajo financiado por la Secretaría de Ciencia y Técnica de la Universidad Nacional del Nordeste. Endereço para correspondência: Lic. María Esther Bar, 9 de Julio 1449. FACENA, UNNE. 3400 Corrientes, Argentina. Tel: + 54-783-24606. Fax: 0054-783-23968. E-mail: mebar @ unefcen.edu.ar Recebido para publicação em 02/07/97.
} 
La frecuencia y gravedad de la enfermedad de Chagas en América varía según la región geográfica19.

Los vectores que transmiten la enfermedad se distribuyen desde los $42^{\circ}$ de latitud norte hasta los $46^{\circ}$ de latitud sur, donde las condiciones ecológicas son propicias para la transmisión y persistencia de la endemicidad18.

Los estudios epidemiológicos ofrecen información fundamental sobre la evolución y patogenia de la cardiopatía provocada por la enfermedad de Chagas. Numerosos reportes asocian clásicamente la infección chagásica a diferentes tipos de alteraciones a nivel del aparato cardiovascular.

Existen diferencias entre las formas clínicas de diversas áreas geográficas, así como en la incidencia de cardiopatía chagásica. Sin embargo, hay investigaciones que no muestran una mayor prevalencia de cardiopatía entre infectados y no infectados. Estos hallazgos plantean la necesidad de intensificar estudios sobre los factores que podrían explicar la variabilidad de las formas clínicas, por ejemplo, el tipo y virulencia de la cepa del T. cruzi y el papel que desempeñan.

De las investigaciones llevadas a cabo en Latinoamérica, en Rio Grande do Sul (Brasil) y en Chile no se encontraron diferencias estadísticamente significativas en la prevalencia de cardiopatías de infectados y no infectados chagásicos 36 18. Asimismo en Venezuela fue verificada la presencia de cardiopatía crónica rural, independiente de la enfermedad de Chagas15. En Chaco, Argentina se observó una prevalencia similar en las alteraciones electrocardiográficas de individuos con serología positiva y los respectivos controles ${ }^{14}$. Los antecedentes sobre el tema son escasos en la provincia de Corrientes, pudiendo citarse los trabajos realizados en los Departamentos de Monte Caseros 17 e Ituzaingó5.

La presente contribución tiene por objeto analizar las características epidemiológicas, clínicas, electrocardiográficas y radiológicas de pobladores seropositivos y seronegativos al $T$. cruzi, en una área rural del Departamento San Miguel.

\section{MATERIAL Y METODOS}

Durante el año 1993 se evaluaron serologicamente 388 pobladores rurales cuyas edades oscilaban entre 0 y 90 años, que habitaban en 100 viviendas precarias de las colonias San Antonio y Caimán, Departamento San Miguel, provincia de Corrientes, Argentina (Figura 1). Las viviendas colonizadas por Triatoma infestans fueron aquellas en las que se detectaron diferentes estados de desarrollo de

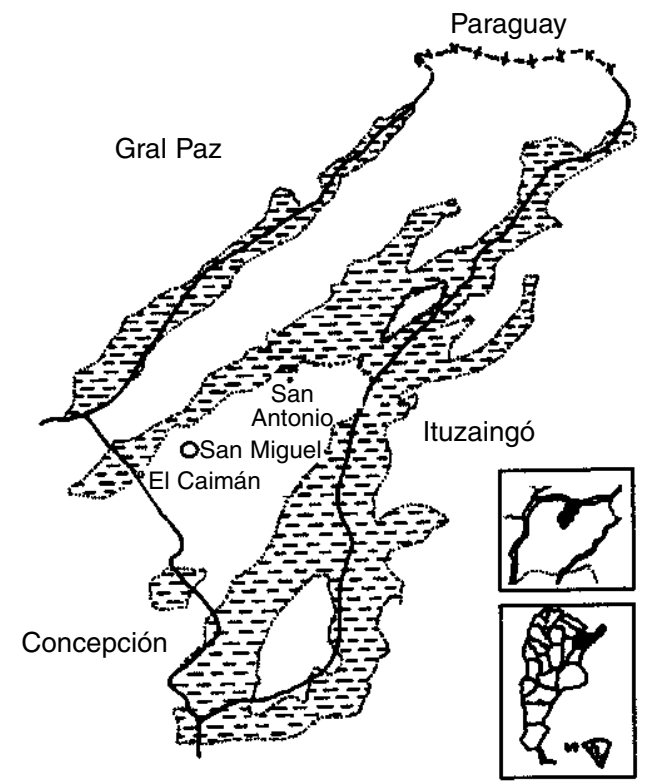
Figura 1 - Localización de las comunidades estudiadas. Departamento San Miguel, provincia de Corrientes, Argentina,
1993. 
la especie, los cuales fueron colectados por el método captura/hora/hombre. Para la evaluación serológica se extrajo sangre por punción venosa. Las muestras de suero fueron conservadas en freezer $\left(-20^{\circ} \mathrm{C}\right)$ hasta su procesamiento. Las técnicas inmunológicas utilizadas para el diagnóstico de la infección chagásica fueron las pruebas de hemaglutinación indirecta e inmunofluorescencia indirecta, considerándose positivos los pacientes con títulos $(\geq 1 / 32$ en los dos test. El $23,4 \%$ de los sueros $(n=91)$ resultaron reactivos para Chagas?

Para la realización de estudios clínicos, con especial énfasis en el aparato cardiovascular, se seleccionaron de la citada población $(n=388)$, por un muestreo simple al azar 132 pobladores de ambos sexos cuyas edades oscilaron entre 2 y 79 años.

Se investigaron síntomas y signos compatibles con afección cardíaca, para lo cual se interrogó sobre antecedentes previos, tales como: síncope, mareos, disnea, dolor precordial, palpitaciones, entre otros. Las edades de los 58 hombres y 74 mujeres oscilaron entre 2 y 79 años, y la edad promedio fue de 31,9 años.

La tensión arterial se controló con un esfingnomanómetro de mercurio, en ambos brazos, promediándose los registros obtenidos.

El electrocardiograma (ECG) de 12 derivaciones, fue registrado a una velocidad de
$25 \mathrm{~mm} / \mathrm{seg}$ con un electrocardiógrafo Fukuda Denshi Cardimax, modelo FK 12. Las alteraciones del electrocardiograma, fueron definidas según Tranchesi23.

A 128 habitantes se les realizó una teleradiografía de tórax (anteroposterior) con un equipo Siemens 100 Miliampere, con placas de $35 \times 35 \mathrm{~cm}$. Los criterios de normalidad y anormalidad radiológica fueron considerados según Cooley7. Los pacientes fueron clasificados de acuerdo a normas del Consejo Argentino de enfermedad de Chagas de la Sociedad Argentina de Cardiología22 en tres grupos, GI: Serología positiva y estudios normales, Gll: Electrocardiograma anormal sin cardiomegalia, GIII: Con cardiomegalia radiológica.

En el análisis estadístico de los datos se aplicaron las pruebas del $\mathrm{x}^{2} \mathrm{y} t$ de Student a un nivel de significación de $\alpha=0,05$.

\section{RESULTADOS}

De los 388 individuos estudiados serologicamente se detectaron 91 con serología positiva para Chagas. En la Tabla 1 se discriminan según grupos etareos y sexo.

Epidemiología. En la muestra objeto del presente estudio $(n=132)$ se constataron 56 $(42,4 \%)$ seropositivos y $76(57,5 \%)$. seronegativos

Tabla 1 - Población examinada serologicamente discriminada según sexo y grupos etareos. Departamento San Miguel. Corrientes, Argentina. 1993.

\begin{tabular}{|c|c|c|c|c|c|c|c|}
\hline \multirow[b]{2}{*}{ Edad } & \multicolumn{3}{|c|}{ Población examinada } & \multicolumn{3}{|c|}{ Seropositivos } & \multirow[t]{2}{*}{$\%$} \\
\hline & femenina & masculina & total & femenino & masculino & total & \\
\hline $2-10$ & 55 & 61 & 116 & 11 & 4 & 15 & 12,9 \\
\hline $11-20$ & 48 & 49 & 97 & 10 & 7 & 17 & 17,5 \\
\hline $21-30$ & 21 & 16 & 37 & 4 & 5 & 9 & 24,3 \\
\hline $31-40$ & 34 & 18 & 52 & 11 & 6 & 17 & 32,7 \\
\hline $41-50$ & 14 & 17 & 31 & 7 & 5 & 12 & 38,7 \\
\hline $51-60$ & 14 & 13 & 27 & 6 & 3 & 9 & 33,3 \\
\hline $61-70$ & 5 & 13 & 18 & 2 & 5 & 7 & 38,9 \\
\hline 71-79 & 6 & 4 & 10 & 4 & 1 & 5 & 50,0 \\
\hline Total & 197 & 191 & 388 & 55 & 36 & 91 & 23,4 \\
\hline
\end{tabular}

En viviendas colonizadas por $T$. infestans habitaban $47,0 \%$ de seropositivos y $53,0 \%$ de seronegativos, mientras que en viviendas sin triatominos vivían $34,0 \%$ de seropositivos y $66,0 \%$ de seronegativos $\left(x^{2}=2,10 ; p=0,20\right)$.

Por otra parte es necesario acotar que un $10 \%$ de la población analizada manifestó haber migrado a áreas de diferente endemicidad chagásica (provincias de Chaco, Santa Fe, diferentes comunidades del Departamento San Miguel etc.).

Signos y síntomas. Los signos y síntomas verificados en los habitantes se presentan en la Tabla 2. Se comparó su prevalencia según serología, no encontrándose diferencias significativas entre seropositivos y seronegativos. 
Revista da Sociedade Brasileira de Medicina Tropical 31:199-206, mar-abr, 1998.

Tabla 2 - Signo-sintomatología de pacientes seropositivos y seronegativos al T. cruzi detectados en el Departamento San Miguel. Corrientes, Argentina. 1993.

\begin{tabular}{lccccc}
\hline Signos y síntomas & Seropositivos & $\%$ & Seronegativos & $\%$ & Valor de P \\
\hline Soplo cardíaco & $12 / 56$ & 21,4 & $12 / 76$ & 15,7 & 0,55 \\
Mareos & $8 / 56$ & 14,2 & $8 / 76$ & 10,5 & 0,70 \\
Disnea & $4 / 56$ & 7,1 & $5 / 76$ & 6,5 & 0,99 \\
Dolor precordial & $8 / 56$ & 14,2 & $12 / 76$ & 15,7 & 0,99 \\
Cefaleas & $3 / 56$ & 5,3 & $3 / 76$ & 3,9 & 0,99 \\
Palpitaciones & $3 / 56$ & 5,3 & $3 / 76$ & 3,9 & 0,99 \\
Síncope & $1 / 56$ & 1,7 & $1 / 76$ & 1,3 & 0,99 \\
\hline
\end{tabular}

Entre los pobladores infectados y con anormalidades electrocardiográficas, menores de 40 años $(n=9)$, se verificó que el $34,6 \%$ presentó un síntoma o signo y en el $44,4 \%$ de éstos se observaron 2 o más. En el grupo mayor de 40 años $(n=14)$, el $53,8 \%$ tenía por lo menos un síntoma, comprobándose en un $71,4 \%$ de éstos últimos 2 o más síntomas.

Electrocardiograma. Se observaron anormalidades electrocardiográficas en 56 (42,4\%) pacientes de los cuales $26(46,4 \%)$ eran seropositivos, y $30(53,6 \%)$ seronegativos. En la Tabla 3 se señalan los electrocardiogramas anormales según edad y sexo. Los trastornos electrocardiográficos discriminados por serología se presentan en la Tabla 4, no verificándose diferencias significativas entre ambos grupos.

Al analizar las anormalidades electrocardiográficas y su relación con los hallazgos clínicos y radiológicos en pacientes

Tabla 3 - Electrocardiogramas anormales según serología, edad y sexo. Departamento San Miguel. Corrientes, Argentina, 1993.

\begin{tabular}{|c|c|c|c|c|c|}
\hline \multirow[b]{3}{*}{ Edad } & \multirow[b]{3}{*}{ ECG trazados } & \multicolumn{4}{|c|}{ Electrocardiogramas anormales } \\
\hline & & \multicolumn{2}{|c|}{ seropositivos } & \multicolumn{2}{|c|}{ seronegativos } \\
\hline & & femenino & masculino & femenino & masculino \\
\hline $2-10$ & 29 & 1 & - & 3 & 3 \\
\hline $11-20$ & 20 & 1 & - & - & 1 \\
\hline $21-30$ & 16 & 2 & 2 & 2 & - \\
\hline $31-40$ & 22 & 4 & 1 & 2 & 1 \\
\hline $41-50$ & 16 & 3 & 2 & 1 & 4 \\
\hline $51-60$ & 14 & 3 & 2 & 2 & 3 \\
\hline $61-70$ & 10 & - & 1 & 2 & 4 \\
\hline $71-79$ & 5 & 3 & 1 & 1 & 1 \\
\hline Total & 132 & 17 & 9 & 13 & 17 \\
\hline
\end{tabular}

Tabla 4 - Tipo de alteraciones ECG presentes en 26 pacientes infectados y 30 no infectados chagásicos. Departamento San Miguel, Corrientes, Argentina, 1993.

\begin{tabular}{|c|c|c|c|c|c|}
\hline \multirow[t]{2}{*}{ Tipo de alteración ECG } & \multicolumn{2}{|c|}{ Seropositivos } & \multicolumn{2}{|c|}{ Seronegativos } & \multirow[t]{2}{*}{ Valor de $\mathrm{P}$} \\
\hline & $\mathrm{n}^{\circ}$ & $\%$ & $\mathrm{n}^{\circ}$ & $\%$ & \\
\hline Sobrecarga de VI & 11 & 42,0 & 14 & 47,0 & 0,95 \\
\hline Sobrecarga de Al & 10 & 38,0 & 9 & 30,0 & 0,70 \\
\hline Extrasístole ventricular & 4 & 15,3 & 1 & 3,3 & 0,27 \\
\hline $\mathrm{HBAI}$ & 3 & 11,5 & 5 & 17,0 & 0,87 \\
\hline BIRD & 3 & 11,5 & 8 & 27,0 & 0,28 \\
\hline BCRD & - & - & 3 & 10,0 & 0,29 \\
\hline Onda Q patológica & 3 & 11,5 & - & - & 0,19 \\
\hline Trastornos de repolarización & 1 & 4,0 & - & - & 0,94 \\
\hline Onda delta & 1 & 4,0 & - & - & 0,94 \\
\hline Eje desviado a la izquierda & 1 & 4,0 & - & - & 0,94 \\
\hline Sobrecarga de cav. derechas & - & - & 1 & 3,3 & 1,00 \\
\hline
\end{tabular}

VI: ventrículo izquierdo, Al: aurícula izquierda, HBAI: hemibloqueo anterior izquierdo, BIRD: bloqueo incompleto de rama derecha, BCRD: bloqueo completo de rama derecha. 
seropositivos, se observó que solamente en 4 de ellos no se detectó otra alteración cardiovascular que pudiera justificar dichas anomalías electrocardiográficas; mientras que en los 22 restantes se registraron cardiopatías asociadas, además de la afección chagásica y que clásicamente se relacionan con dichas anormalidades electrocardiográficas. Las cardiopatías coexistentes fueron: miocardiopatía hipertrófica en 1, hipertensión arterial en 13, síndrome de Wolff Parkinson White en 1, secuela de infarto en 2, valvulopatías mitrales y aórticas en 5 .

En 12 pacientes con ECG anormal y serología negativa no se encontraron otras afecciones cardíacas concomitantes, mientras que en los 18 restantes se constataron cardiopatías coexistentes, tales como: hipertensión arterial en 9, valvulopatías mitrales y aórticas en 7 , secuela de infarto en 1 , y una no fue identificada con los métodos empleados.

El $21,4 \%$ de las mujeres y el $16,1 \%$ de los varones con ECG anormal tenían antecedentes o se les registró hipertensión arterial al momento del examen.
Si bien los pobladores seropositivos al T. cruzi y ECG anormal vivían en mayor número en viviendas colonizadas por $T$. infestans $(n=17)$ que en viviendas sin infestación $(n=9)$ no se observaron diferencias significativas $\left(x^{2}=1,35 ; p=0,25\right)$, mientras que los seronegativos con ECG anormal habitaban en igual proporción en viviendas infestadas y no infestadas por $T$. infestans $(n=15)$.

Radiografía de tórax. De las 128 radiografías de tórax realizadas, se visualizaron anormalidades en un $29,6 \%$, dichas alteraciones se detallan en la Tabla 5 y se cuantifican según edad y sexo en la Tabla 6.

De la población seropositiva, ninguno fue clasificado en el GIII ya que los 3 pacientes con cardiomegalia eran seronegativos. El 53,8\% de los pacientes pertenecían al GI, cuya edad media fue de 20,6 años y el $46,2 \%$ restante fue categorizado en el GII, con edad media de 45,0 años. Se observaron diferencias altamente significativas entre las edades de los GI y II $(t=4,96 ; p=0,00001)$.

Tabla 5 - Anomalías detectadas por radiografías de tórax. Departamento San Miguel. Corrientes, Argentina. 1993.

\begin{tabular}{|c|c|c|c|}
\hline \multirow[b]{2}{*}{ Tipo de alteración } & \multicolumn{3}{|c|}{ Radiografías } \\
\hline & pacientes infectados & pacientes no infectados & total \\
\hline \multicolumn{4}{|l|}{ Vasos } \\
\hline Aorta prominente & 13 & 19 & 32 \\
\hline Arco medio prominente & 3 & 1 & 4 \\
\hline \multicolumn{4}{|l|}{ Corazón } \\
\hline Cardiomegalia global & 0 & 3 & 3 \\
\hline Agrandamiento del VI & 0 & 1 & 1 \\
\hline Total & 16 & 24 & $40^{*}$ \\
\hline
\end{tabular}

*En dos pacientes no infectados se presentaron alteraciones tanto en vasos como en corazón. VI: ventrículo izquierdo.

Tabla 6 - Radiografías con alteraciones de seropositivos y seronegativos discriminadas según edad y sexo. Departamento San Miguel, Corrientes, Argentina. 1993.

\begin{tabular}{|c|c|c|c|c|c|}
\hline \multirow[b]{3}{*}{ Edad } & \multirow[b]{3}{*}{ Radiografías realizadas } & \multicolumn{4}{|c|}{ Radiografías anormales } \\
\hline & & \multicolumn{2}{|c|}{ seropositivos } & \multicolumn{2}{|c|}{ seronegativos } \\
\hline & & femenino & masculino & femenino & masculino \\
\hline $2-10$ & 25 & 1 & - & - & - \\
\hline $11-20$ & 21 & - & - & 1 & - \\
\hline $21-30$ & 16 & 2 & - & 1 & 1 \\
\hline $31-40$ & 23 & 1 & - & 3 & 3 \\
\hline $41-50$ & 13 & 1 & 1 & 4 & 1 \\
\hline $51-60$ & 16 & 3 & 2 & 2 & 1 \\
\hline $61-70$ & 8 & - & 2 & 1 & 2 \\
\hline $71-79$ & 6 & 2 & 1 & 1 & 1 \\
\hline Total & 128 & 10 & 6 & 13 & 9 \\
\hline
\end{tabular}




\section{DISCUSION}

La población estudiada no muestra diferencias en la prevalencia de alteraciones cardiovasculares: clínicas, electrocardiográficas ni radiológicas, entre los individuos con serología positiva y los de serología negativa .

Del análisis de los datos obtenidos en el interrogatorio y el examen físico-cardiovascular surge que si bien se observa una mayor prevalencia de dolor precordial en los seronegativos, la diferencia no es significativa estadísticamente. El resto de los síntomas analizados están presentes con similar frecuencia en ambos grupos.

La principal alteración electrocardiográfica de los infectados chagásicos es la sobrecarga de cavidades izquierdas, valores menores fueron hallados en reportes previos 410 . Las arritmias ventriculares son frecuentemente observadas en estos pacientes 20 , los valores encontrados en este estudio son semejantes a los citados en trabajos anteriores 81016 y cifras menores son presentadas por otros autores 911121324 . Indudablemente la prevalencia de arritmias ventriculares depende principalmente de las características clínicas de la población estudiada y del método utilizado para detectarlas. Cabría esperar que en la presente investigación, dado el importante número de anormalidades cardiovasculares detectadas, se haya subestimado la real prevalencia de arritmias, al no utilizarse una técnica de monitoreo continuo tipo Holter.

Si bien el bloqueo de rama derecha completo o incompleto es la alteración eletrocardiográfica, más frecuentemente, observada en pacientes chagásicos $1912 \quad 162021$ 22, en el presente estudio ningún paciente infectado presentó BCRD , sin embargo se detectó BIRD en cifra semejante al obtenido por Dávila et al9.

EI HBAI, trastorno electrocardiográfico que le sigue en importancia al BCRD entre los pacientes seropositivos, ha sido confirmado entre un $4,0 \%$ y $8,5 \% 81322$, el resultado hallado en el presente aporte es similar al constatado por otros autores 91620.

La baja proporción de trastornos de repolarización inespecíficos registrados coinciden con los hallados por otros investigadores 11622 , valores mayores (hasta 19,4\%) son citados en series previas 1013 . Esta divergencia podría deberse a la disparidad de criterios para considerar esta anormalidad electrocardiográfica.
En los seronegativos las sobrecargas ventriculares y auriculares izquierdas, se presentan en porcentajes menores a los obtenidos previamente 4 .

EI BIRD alcanza una cifra mayor que en los seropositivos y menor que las citadas por otros autores 14.

El HBAl en seronegativos se presenta en porcentaje menor que el confirmado por Arribada et al1 y mayor a los citados en publicaciones anteriores 816 .

EI BCRD detectado sólo en seronegativos, alcanza un valor semejante al observado en una serie previa ${ }^{1}$ y menor que los mencionados en otros reportes 416.

Cabe destacar que una proporción elevada de los pobladores con alteraciones electrocardiográficas son hipertensos $(37,5 \%)$.

Se concluye que los diferentes tipos de alteraciones electrocardiográficas y radiográficas verificados tanto en pacientes seropositivos como seronegativos, al igual que la detección de signos y síntomas, indicarían que el origen de las mismas no serían estrictamente adjudicables a la enfermedad de Chagas, dada la presencia de otras afecciones que prevalecen en el área de estudio y que tienen similar manifestación cardíaca.

Un análisis más exhaustivo de los resultados plantea las siguientes consideraciones:

- La población chagásica evaluada pertenecía a un grupo etareo más joven que el que habitualmente desarrolla la miocardiopatía chagásica (mayor de 45 años) dado que el promedio de edad era de 31,9 años y sólo el $36,0 \%(33 / 91)$ superaba los 41 años; perteneciendo casi el $54,0 \%$ de los pacientes al GI con una edad inferior a 20 años.

De los 26 ECG anormales hallados en la población chagásica, 15 de ellos, es decir el $58,0 \%$, pertenecían a pacientes de más de 41 años. Además, el 45,0\% (15/33) de los pacientes mayores de esta edad presentaron ECG con alteraciones, hallazgo similar al de otros autores 101221.

- Con respecto a la población no chagásica, debe destacarse que el $30,0 \%$ de los ECG patológicos se presentaron en individuos menores de 30 años por lo que no debería descartarse la presencia de cardiopatías congénitas (más aún considerando la frecuencia 
del hallazgo de soplos cardíacos) que pudieron haber influido en los resultados.

- En ambos grupos se observó una importante prevalencia (más de 60,0\%) de cardiopatías asociadas, especialmente hipertensión arterial, por lo que no debe descartarse que estas alteraciones equiparen en alguna medida las muestras y enmascaren los resultados.

Asimismo, se asumen las limitaciones del presente estudio de corte transversal, representadas principalmente por el tamaño de la muestra y las técnicas empleadas para evaluar las alteraciones.

Por lo antes expresado se plantea como tema de futuras investigaciones el seguimiento longitudinal de una cohorte de pacientes que permitan verificar la evolución temporal de los mismos, así como realizar estudios sobre el aislamiento del $T$. cruzi presente en la región (caracterización de la cepa y virulencia) que pudieran validar los hallazgos del presente aporte.

\section{AGRADECIMIENTOS}

Expresamos nuestra gratitud por la valiosa colaboración prestada a los Doctores R.J. Badaracco, Director del Instituto de Cardiología de Corrientes "Juana F. Cabral" y P. Ortiz, Director del Hospital de San Miguel; Supervisor y Agentes de Salud Rural del Departamento San Miguel; Estadístico E. Porcel; Lic. M. Damborsky, Lic. E. Oscherov, Prof. G. Avalos y Bioquímica M.H. Alegre y al Dr. S. Sosa Estani por la lectura crítica del manuscrito .

\section{REFERENCIAS BIBLIOGRÁFICAS}

1. Arribada $A C$, Werner $A B$, Ugarte JM. Evolución de la cardiopatía chagásica durante un período de cuatro años en un grupo de pacientes chilenos. Boletín de la Oficina Sanitaria Panamericana 102: 49-72, 1987.

2. Bar ME, Oscherov EB, Damborsky MP, Alvarez BM, Mizdraji G, Avalos G, Porcel E. Infestación doméstica por Triatoma infestans y prevalencia de seropositivos al Trypanosoma cruzi en una área rural del Nordeste Argentino. Revista da Sociedade Brasileira de Medicina Tropical 29:549-555,1996.

3. Baruffa G, Aquino Neto JO, Alcantara Filho A, Bettini VN, Bertinetti ES. Dados preliminares de inquérito sorológico e eletrocardiográfico para doença de Chagas em populações rurais não selecionadas da zona sul do Rio Grande do Sul. Revista da Sociedade Brasileira de Medicina Tropical 6:362-
368,1972.

4. Bonet AH, Cichero JA, Kuschnir E, Grand JC, Segura EL. Estudio epidemiológico sobre la enfermedad de Chagas Mazza en comunidades rurales de la provincia de Córdoba. Encuesta serológica y electrocardiográfica. Semana Médica 133:581-614, 1968.

5. Borda CE, Rea MJF, Badaracco JR, Pozzer DL. Estudio de la Enfermedad de Chagas en la Isla Apipé Chico (Corrientes, Argentina). II Clínica y electrocardiografía. In: Resumen IX Congreso Latinoamericano de Parasitología, Caracas, Venezuela p. 234, 1989.

6. Brandt TC, Laranja FS, Leite Mello A. Dados sorológicos e eletrocardiográficos obtidos em populações não selecionadas de zonas endêmicas de doença de Chagas no Estado do Rio Grande do Sul. Revista Brasileira de Malariologia e Doenças Tropicais 19: 141-148, 1957.

7. Cooley R, Schreiber M. Radiografía de corazón y grandes vasos. Editorial Panamericana, $3^{\mathrm{a}}$ edición, Buenos Aires, 1980.

8. Coura RJ, Abreu LL, Dubois LEG, Correia-Lima F, Arruda Jr ER, Willcox HPF, Anunziato N, Petana W. Morbidade da doença de Chagas. II-Estudos seccionais em quatro áreas de campo no Brasil. Memórias do Instituto Oswaldo Cruz Rio de Janeiro 79: 101-124, 1984.

9. Dávila H, Beloscar JS, Bottasso OA, Morini JC. Alteraciones electrocardiográficas en individuos infectados con Trypanosoma cruzi con distinto tiempo de residencia en áreas de alta endemicidad. Medicina (Buenos Aires) 147:154-158, 1987.

10. Dias JCP. Enfermedad de Chagas. EpidemiologíaClínica-Terapeutica. Programa de Salud Humana, Buenos Aires, 1984.

11. Goldsmith RS, Zárate RJ, Zárate LG, Morales G, Kagan I, Drickey R, Jacobson LB. Estudios clínicos y epidemiológicos sobre la enfermedad de Chagas en comunidades rurales de Oaxaca, México; un estudio de ocho años de seguimiento: II. Chila. Boletín de la Oficina Sanitaria Panamericana 113: 97-108, 1992.

12. Laranja FS, Dias E, Nobrega G, Miranda A. Chagas' disease. A clinical, epidemiologic and pathologic study. Circulation 14: 1035-1060, 1956.

13. Libonatti EJ, Mazullo EC, Darraidou MA, Quiñones G, Morgade S, Libonatti OV. Seguimiento longitudinal de 4.441 chagásicos crónicos en nueve años. Revista Argentina de Enfermedades Transmisibles 1: 35-48, 1980.

14. Mendivil GT, Finkielman S, Gorodner JO, 
Bustamante A, Guilleron CD, Tercelán R, Gatti G, González R. Evidencia epidemiológica de enfermedad cardíaca no relacionada con el Trypanosoma cruzi en zonas endémicas para la enfermedad de Chagas. Medicina (Buenos Aires) 44: 489-494, 1984.

15. Montero DN, Ross A, Torres RA, Nava Rodríguez M, Hernández Nieto RA. Cardiopatía crónica endémica rural venezolana - Fenómeno independiente de la enfermedad de Chagas crónica. Trujillo, Venezuela, 1978-1994. Conferencia. In: VIII Jornadas Capítulo Merideño de Medicina Interna, Mérida, p. 1-17, 1994.

16. Morini JC, Berra H, Dávila HO, Pividori JF, Bottasso OA. Electrocardiographic alteration among first degree relatives with serologic evidence of Trypanosoma cruzi infection. A sibship study. Memórias do Instituto Oswaldo Cruz. Rio de Janeiro 89: 371-375, 1994.

17. Mujica LP, Fontana LE, Ruso MC, Alvarez M. Encuesta serológica y electrocardiográfica en la población del Departamento Monte Caseros, Corrientes. Medicina (Buenos Aires) 40 (supl 1): 251 252, 1980.

18. Organización Mundial de la Salud-Oficina Sanitaria Panamericana. Aspectos clínicos de la enfermedad de Chagas. Informe de una reunión conjunta OMS/OPS de investigadores. Boletin de la Oficina Sanitaria Panamericana 76:141-158, 1974.
19. Organización Mundial de la Salud. Memoranda: Inmunology of Chagas' disease. Bulletin World Health Organization 50:459-472, 1974.

20. Pereira AG, Calegari AM. Miocardiopatía chagásica crónica. Revista Médica del Uruguay 2: 186-192, 1986.

21. Rosenbaum MB, Alvarez AJ. The electrocardiogram in chronic chagasic myocarditis. American Heart Journal 50: 492-527, 1955.

22. Storino R, Milei J. Estudios de seguimiento evolutivo de la enfermedad de Chagas. In: Madoery RJ, Madoery C, Cámera MI (eds). Actualizaciones en la enfermedad de Chagas, Córdoba, p. 67-77, 1993.

23. Tranchesi J. Electrocardiograma normal y patológico. Nociones de vectocardiografía. Beta S.R.L, 4ae edición, Uruguay, 1987.

24. Wisnivesky-Colli C, Ruiz AM, Gürtler RE, Solarz ND, Lazzari J, Ledesma O, Bujas MA, Rissio AM, Marteleur A, Segura EL. Dynamic of transmission of Trypanosoma cruzi in a rural area of Argentina. IV. Serologic, parasitologic and electrocardiographic study of the human population. Medicina (Buenos Aires) 49: 341-350, 1989. 